Panorama ECONómico, Volumen IV, No. 8, enero-junio, 2009, pp. 63-100

\title{
ANCLAS NOMINALES Y SU IMPACTO EN ECONOMÍAS PEQUEÑAS: UN ANÁLISIS COMPARATIVO ENTRE LOS ESQUEMAS DETERMINISTA Y ESTOCÁSTICO
}

\author{
Francisco Venegas-Martínez* \\ Abigail Rodríguez Nava**
}

\begin{abstract}
RESUMEN
En este trabajo se desarrollan varios modelos para estudiar, en los marcos determinista y estocástico, las políticas de estabilización de precios que utilizan al tipo de cambio como un ancla nominal. Los modelos propuestos permiten examinar los efectos de estas políticas en la cuenta corriente de la balanza de pagos, el tipo de cambio real y el consumo. En el marco determinista se muestra que estos programas de estabilización generan un déficit temporal sobre la cuenta corriente de la balanza de pagos en economías con un bien (comerciable internacionalmente) y una apreciación del tipo de cambio real en economías con dos bienes (comerciables y no comerciables). En el primer caso (con un bien), entre más corto sea el periodo de estabilización, mayor será el déficit

* Profesor-Investigador de la Sección de Estudios de Posgrado e Investigación de la Escuela Superior de Economía, Instituto Politécnico Nacional. Miembro del Sistema Nacional de Investigadores (SNI III). Correo electrónico: <fvenegas@ipn.mx>.

** Profesora-Investigadora de la Universidad Autónoma Metropolitana, Unidad Xochimilco. Miembro del Sistema Nacional de Investigadores (SNI C). Correo electrónico: <nava11@prodigy.net.mx>.
\end{abstract}


en la cuenta corriente. En el segundo caso (con dos bienes), entre más corto sea el periodo de estabilización, mayor será la apreciación del tipo de cambio real. Posteriormente, se desarrolla un modelo estocástico de estabilización temporal con supuestos similares al marco determinista para estudiar el comportamiento del consumo. Por último, se analizan las diferencias entre los casos determinista y estocástico y se establecen sus ventajas y limitaciones, en términos de los resultados que proporcionan.

Palabras clave: Estabilización de precios, tipo de cambio, política macroeconómica

Clasificación JEL: F31, F41, E6

\begin{abstract}
This paper is aimed to develop several models to study stabilization policies under the deterministic and stochastic frameworks when the exchange rate is taken as a nominal anchor. The proposed models are useful to examine the effects of such policies on the current account of the balance of payments, the real exchange rate, and consumption. Under the deterministic scheme, it is shown that this kind of stabilization programs generates a temporary deficit on the current account of the balance of payments in economies with one good (internationally tradable), and an appreciation of the real exchange rate in economies with two goods (tradable and non tradable). In the first case (with a single good) the shorter the stabilization period, the greater the deficit in the current account. In the second case (with two goods) the shorter the stabilization period, the greater the type of real exchange rate. Subsequently, a stochastic model of temporary stabilization is developed under similar assumptions to the deterministic scheme to study consumption behavior. Finally, the deterministic and stochastic frameworks are compared and their advantages and limitations are established in terms of the provided results.

Keywords: Inflation stabilization, exchange rate, macroeconomic policy JEL classification: F31, F41, E6
\end{abstract}


ANCLAS NOMINALES Y SU IMPACTO EN ECONOMÍAS PEQUEÑAS: UN ANÁLISIS COMPARATIVO ENTRE LOS ESQUEMAS DETERMINISTA Y ESTOCÁSTICO

\section{INTRODUCCIÓN}

Mucho se ha aprendido sobre los programas de estabilización de precios que utilizan el tipo de cambio como un ancla nominal. Los casos de México (1994), Brasil (1999) y Argentina (2001) han proporcionado abundante información que ha sido examinada ampliamente en la literatura especializada (véanse, por ejemplo, las referencias en Calvo y Végh, 1999 y Venegas-Martínez, 2001). Pero, también mucho queda por aprender sobre sus efectos en la cuenta corriente de la balanza de pagos, el tipo de cambio real, el consumo y las decisiones de portafolio de los agentes. También es importante mencionar que, de acuerdo con el Fondo Monetario Internacional (FMI), actualmente más del 10\% de los países en el mundo tienen regímenes cambiarios que utilizan al tipo de cambio como un ancla nominal.

Uno de los principales objetivos de este trabajo es proporcionar, con base en modelos teóricos deterministas y estocásticos, la explicación de algunas de las causas por las que han fallado estos intentos de estabilización en el corto y mediano plazos y analizar el impacto de estos fracasos en variables macroeconómicas fundamentales. Es importante mencionar que esta investigación extiende los trabajos de Drazen y Helpman (1988) quienes analizan un programa de estabilización con un tipo de cambio estocástico y Uribe (2002) y Uribe y Mendoza (2000) quienes analizan los órdenes de magnitud de los booms inesperados de consumo y la incorporación de la incertidumbre en el análisis de los planes de estabilización temporal.

En este trabajo de investigación se analiza, primero con un modelo determinista, el impacto que sobre la cuenta corriente y el tipo de cambio real tiene una política de estabilización basada en una disminución temporal de la tasa de devaluación. Para ello, se considera una economía poblada por consumidores con preferencias y dotaciones idénticas, cada uno de los cuales viven para siempre y desea maximizar su satisfacción por un bien genérico de consumo. Asimismo, se supone que estos agentes tienen expectativas racionales (previsión perfecta), es decir, conocen en cada instante el nivel general de precios. Los supuestos iniciales serán al principio 
muy simples, de tal manera que el modelo sea manejable y que, al mismo tiempo, capture los efectos importantes en variables relevantes (cuenta corriente, tipo de cambio real, etc.). En una segunda etapa se modifican o se extienden los supuestos y se examina si los resultados antes obtenidos siguen siendo válidos. Posteriormente, se desarrolla un modelo estocástico de estabilización temporal y sus resultados son comparados con los del caso determinista.

El presente trabajo está organizado de la siguiente manera. En la siguiente sección se enlistan los supuestos iniciales del modelo y se proponen varias modificaciones y extensiones más realistas a dichos supuestos. En la sección 3 se presenta un modelo determinista básico de estabilización temporal. En la sección 4 se modifica y extiende el modelo planteado en la sección anterior. En el transcurso de la sección 5 se extienden los modelos anteriores a un ambiente de riesgo e incertidumbre. En la sección 6 se presentan las conclusiones, así como las limitaciones y sugerencias para futuras investigaciones. Por último, se presentan tres apéndices para las secciones 3, 4 y 5 sobre la equivalencia de los problemas de decisión y algunos detalles técnicos de varios resultados analíticos.

\section{SUPUESTOS DEL MODELO}

En esta sección se describen, brevemente, los supuestos relevantes del modelo básico de estabilización temporal de precios que toma como ancla nominal el tipo de cambio. Dichos supuestos se enlistan a continuación:

i. la economía es pequeña (tomadora de precios),

ii. la economía produce y consume un bien (genérico) comerciable (internacionalmente),

iii. existen dos activos, moneda nacional y un bono internacional,

iv. los agentes tienen acceso a un mercado internacional de crédito, v. existe perfecta movilidad de capital, 
ANCLAS NOMINALES Y SU IMPACTO EN ECONOMÍAS PEQUEÑAS: UN ANÁLISIS COMPARATIVO ENTRE LOS ESQUEMAS DETERMINISTA Y ESTOCÁSTICO

vi. $\quad$ se cumplen las condiciones de paridad de poder de compra y paridad de tasas de interés,

vii. $\quad$ se cumple la condición cash-in-advance (dinero y consumo son perfectos sustitutos),

viii. existe pleno empleo,

ix. los individuos tienen preferencias y dotaciones idénticas,

x. los individuos son consumidores racionales,

xi. los individuos tienen vida infinita,

xii. los agentes tienen previsión perfecta,

xiii. la tasa subjetiva de descuento coincide (por mera casualidad) con la tasa de interés real,

xiv. el resto del mundo no posee dinero doméstico.

Con base en este primer modelo se pretende mostrar que una política de estabilización basada en una disminución temporal de la tasa de devaluación genera un déficit temporal sobre la cuenta corriente. En este caso, entre más corto sea el periodo de estabilización, mayor será el déficit en cuenta corriente que experimenta la economía. Posteriormente, se extenderá el modelo anterior con las siguientes modificaciones a los supuestos y se examinará, en lo que sigue del trabajo, si los resultados obtenidos siguen siendo válidos. Los supuestos que se incorporarán son:

a) inclusión de bienes no-comerciables,

b) el dinero proporciona utilidad por servicios de liquidez, y consumo y dinero son sustitutos a la Edgeworth,

c) saldos reales y consumo no son perfectos sustitutos,

d) no hay movilidad de capital.

\section{EL MODELO BÁSICO}

Considere una economía pequeña y abierta que produce y consume un solo bien y está poblada por consumidores, que viven para siempre, con preferen- 
cias y dotaciones idénticas que desean maximizar su satisfacción por un bien genérico de consumo. Suponga que dicha satisfacción está dada, al tiempo $t=0$, por:

$$
V(0)=\int_{0}^{\infty} u[c(t)] e^{-\rho t} \mathrm{~d} t
$$

donde $c(t)$ es el consumo, $\rho$ es la tasa subjetiva intertemporal de descuento y es la función de utilidad, la cual se supone creciente y estrictamente cóncava con segunda derivada continua y que, además, satisface

Por lo tanto, el índice de felicidad del individuo por el consumo, al tiempo $s>0$, está representado por:

$$
\lim _{c \rightarrow \infty} \partial^{\prime}(c)=0
$$

$$
V(s)=\int_{s}^{\infty} u[c(t)] e^{-\rho(t-s)} \mathrm{d} t
$$

Para ser más específicos se considera una función de utilidad de la forma

$$
c(t)= \begin{cases}\frac{c(t)^{1-\gamma}}{1-\gamma}, & \text { para } \gamma>0, \quad \gamma \neq 1, \\ \log c(t), & \text { para } \gamma=1,\end{cases}
$$

en donde se ha removido la discontinuidad de la función en $\gamma=1$. Observe que, para esta función de consumo la elasticidad de sustitución entre $c(t)$ y $c(s)$ con $s \neq t$ está dada por 
ANCLAS NOMINALES Y SU IMPACTO EN ECONOMÍAS PEQUEÑAS: UN ANÁLISIS COMPARATIVO ENTRE LOS ESQUEMAS DETERMINISTA Y ESTOCÁSTICO

También note que cuando $s \rightarrow t$, la elasticidad de sustitución satisface

En este caso, el grado relativo de aversión al riesgo cumple con

$$
-u^{\prime \prime}[c(t)] \frac{c(t)}{u^{\prime}[c(t)]}=\gamma
$$

Se supone, también, que el individuo representativo tiene previsión perfecta, es

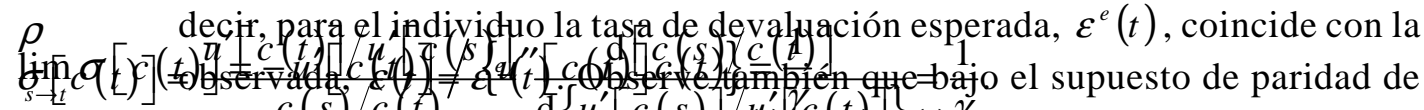
poder de compra se cunple que $P(t)=e(t) p s(t)$, donde $P(t)$ es el precio doméstico, $P^{*}(t)$ es el precio en el resto del mundo (en dólares por bien) y $e(t)$ es el tipo de cambio nominal. Si se utiliza ahora el supuesto de economía pequeña (tomadora de precios), entonces $P^{*}(t)$ es tomado como dado. Sin pérdida de generalidad se puede suponer que $P^{*} \equiv 1$. De esta manera, la tasa de devaluación, $\varepsilon(t)$, coincide con la tasa de inflación, $\pi(t)$, esto es,

$$
\varepsilon(t)=\dot{e}(t) / e(t)=\pi(t)=\dot{P}(t) / P(t) .
$$

Además, se supone que hay perfecta movilidad de capital (específicamente, perfecta movilidad de bonos) y que la tasa real de interés del resto del mundo, $r$, satisface $\rho=r$, esto es, el parámetro de preferencias, coincide (por casualidad) con la tasa de interés, entonces se cumple la siguiente condición de arbitraje 
A continuación, se supone que la riqueza financiera (real) del individuo, $a(t)$, a través del tiempo, está determinada por activos en moneda nacional, en términos de saldos monetarios reales, $m(t)$, y un bono internacional, $F(t)$, de tal manera que

$$
a(t)=m(t)+F(t)
$$

donde $F(0)$ es una variable exógena. Sea y el ingreso que se supone constante en todo momento. Suponga que hay un gobierno, con el mismo horizonte de planeación del agente representativo, que hace transferencias de suma fija (lumpsum), $g(t)$ al individuo. La restricción presupuestal del individuo, una vez que ha incorporado sus expectativas y las transferencias del gobierno, está dada por:

$$
a(0)+\frac{y}{r}+\int_{0}^{\infty} g(t) e^{-r t} \mathrm{~d} t=\int_{0}^{\infty}[c(t)+i(t) m(t)] e^{-r t} \mathrm{~d} t .
$$

Esta condición dice que, para el individuo representativo, el valor presente del flujo de ingresos futuros debe ser igual el valor presente del gasto planeado. La ecuación (4) se puede reescribir en forma alternativa como:

$$
\dot{a}(t)=y+g(t)+r F(t)-c(t)-\varepsilon(t) m(t),
$$

junto con la condición de transversalidad

$$
\lim _{t \rightarrow \infty} a(t) e^{-r t}=0
$$

En efecto, como puede observarse

$$
a(0)-\lim _{t \rightarrow \infty} a(t) e^{-r t}=\int_{0}^{\infty}[r a(t)-a(t)] e^{-r t} \mathrm{~d} t,
$$


ANCLAS NOMINALES Y SU IMPACTO EN ECONOMÍAS PEQUEÑAS: UN ANÁLISIS COMPARATIVO ENTRE LOS ESQUEMAS DETERMINISTA Y ESTOCÁSTICO

con lo cual, a partir de (4), se tiene que

$$
r a(t)-\dot{a}(t)+y+g(t)=c(t)-i(t) m(t)
$$

Después de sustituir (2) y (3) en la expresión anterior se obtiene, finalmente, la ecuación (5). Es importante notar que, bajo el supuesto de perfecta movilidad de capital, la condición no Ponzi, dada en (6), está eliminando la posibilidad de que el individuo se endeude indefinidamente para pagar intereses con más y más deuda.

A continuación se introduce la condición cash-in-advance. En esta condición se establece que el consumo planeado tiene que financiarse con saldos monetarios reales de tal forma que

$$
m(t) \geq \alpha c(t)
$$

El individuo representativo desea determinar la trayectoria de consumo que maximice su utilidad total, $V(0)$, dada en (1), sujeto a las restricciones presupuestal y cash-in-advance, (4) y (9), respectivamente. Es decir, el individuo representativo desea encontrar la trayectoria de consumo que resuelva el siguiente problema:

$$
\begin{gathered}
\text { Maximizar } \int_{0}^{\infty} u[c(t)] e^{-r t} \mathrm{~d} t, \\
\text { sujeto a : }\left\{\begin{array}{l}
r a(t)-\dot{a}(t)+y+g(t)=c(t)-i(t) m(t), \quad a_{0} \text { dado, } \\
m(t)=\alpha c(t) .
\end{array}\right.
\end{gathered}
$$

En cuyo caso el Lagrangiano ${ }^{1}$ del correspondiente problema variacional está dado por

\footnotetext{
1 Ver apéndice a esta sección.
} 


$$
L[c(t), \lambda]=\{u[c(t)]+\lambda\{y+g(t)-c(t)(1+\alpha i(t))\}\} e^{-r t},
$$

donde $\lambda$ es el multiplicador de Lagrange asociado a la restricción (4). Se supone también que la restricción (9) está activa, equivalentemente, se supone que para toda $t$.

Es importante destacar aquí que, si se hubiera llevado a cabo un análisis con una función de utilidad de la forma $U=U[c(t), m(t)]$, entonces al suponer que (9) está activa resultaría que

$$
U=U(c(t), m(t)]=U(c(t), \alpha c(t)]=u[c(t)]
$$

es decir, consumo y saldos reales son perfectos sustitutos. En este caso, los agentes pueden mantener saldos reales, o bien por los servicios de liquidez o para financiar consumo.

Ahora bien la condición de primer orden (condición necesaria de óptimo, ecuación de Euler-Lagrange) del problema de maximización de utilidad resulta ser

$$
\lambda(1+\alpha i(t))=c(t)^{-\gamma}, \text { para todo } \gamma>0 .
$$

Esta condición dice que la utilidad marginal del consumo es igual a su precio multiplicado por el precio sombra de la riqueza. Se entiende que el precio de $c(t)$ es igual a su costo de producción $(=1)$ más el costo de oportunidad de mantener un nivel de saldos monetarios reales $m(t)$. Observe que el precio sombra de la riqueza satisface

$$
0>\frac{\partial \lambda}{\partial c}=-\gamma(t)^{-(\gamma+1)}, \text { para todo } \gamma>0
$$

A continuación se considera la restricción presupuestal del gobierno

$$
g(t)+[\dot{b}(t)-r b(t)]=\dot{m}(t)+\varepsilon(t) m(t)
$$


ANCLAS NOMINALES Y SU IMPACTO EN ECONOMÍAS PEQUEÑAS: UN ANÁLISIS COMPARATIVO ENTRE LOS ESQUEMAS DETERMINISTA Y ESTOCÁSTICO

En este caso también se tiene una condición de transversalidad

$$
\lim _{t \rightarrow \infty} b(t) e^{-r t}=0
$$

ya que

$$
b(0)-\lim _{t \rightarrow \infty} b(t) e^{-r t}=\int_{0}^{\infty}[r b(t)-\dot{b}(t)] e^{-r t} \mathrm{~d} t,
$$

aquí $b(t)$ denota la tenencia de bonos internacionales por parte del gobierno al tiempo $t$ y $\dot{m}(t)+\varepsilon(t) m(t)$ representa el ingreso por impuesto inflacionario (señoriaje) debido a la creación de dinero. Aquí $b(0)$ es exógeno. La ecuación (12) se puede reescribir como

$$
\int_{0}^{\infty} g(t) e^{-r t} \mathrm{~d} t=b(0)+\int_{0}^{\infty}[\dot{m}(t)+\varepsilon(t) m(t)] e^{-r t} \mathrm{~d} t
$$

Si se denota por $B(t)$ el total de bonos internacionales en la economía, se tendrá que:

$$
B(t)=b(t)+F(t)
$$

De la ecuación (3), de la riqueza financiera del individuo representativo, se tiene ahora que

$$
a(t)=m(t)+B(t)-b(t)
$$

Si se utiliza ahora la restricción del flujo de la riqueza (5), se obtiene

$$
\dot{m}(t)+\dot{B}(t)+\dot{b}(t)=y+g(t)+r[B(t)-b(t)]-c(t)-\varepsilon(t) m(t)
$$


Equivalentemente

$$
g(t)+[\dot{b}(t)-r b(t)]-[\dot{m}(t)+\varepsilon(t) m(t)]=[\dot{B}(t)-r B(t)]+c(t)-y,
$$

con lo cual se obtiene que la restricción presupuestal de la economía en su conjunto puede escribirse como:

$$
B(0)+\frac{y}{r}=\int_{0}^{\infty} c(t) e^{-r t} \mathrm{~d} t
$$

Esta ecuación simplemente dice que, para la economía, el valor presente del flujo de ingresos futuros debe ser igual al valor presente del consumo planeado. Observe también que el dinero doméstico no aparece en la restricción (20) ya que éste no representa riqueza para el resto del mundo, pues el resto del mundo no posee moneda nacional.

A continuación se estudian programas de estabilización en los cuales la tasa de devaluación satisface

$$
\varepsilon_{0}= \begin{cases}\mathcal{E}_{0}, & \text { para } 0 \leq t \leq T, \\ \mathcal{E}_{1}, & \text { para } t>T,\end{cases}
$$

donde $\varepsilon_{0}<\varepsilon_{1}$. Para ser consistentes con , es necesario suponer que $\varepsilon_{1}+r>\varepsilon_{0}+r>0$.

Observe que bajo los supuestos de economía pequeña y poder de paridad de compra, tomando unidades adecuadas de tal manera que se mantenga a través del tiempo, se tiene que la tasa de devaluación coincide con la tasa de inflación, $\varepsilon(t)=\dot{p} / p$. Si se utiliza ahora la ecuación (11), se tiene que en el intervalo $[0, T]$

$$
c(t)=\left\{\lambda\left[1+\alpha\left(\varepsilon_{0}+r\right)\right]\right\}^{-\frac{1}{\gamma}}, \text { para todo } \gamma>0
$$


ANCLAS NOMINALES Y SU IMPACTO EN ECONOMÍAS PEQUEÑAS: UN ANÁLISIS COMPARATIVO ENTRE LOS ESQUEMAS DETERMINISTA Y ESTOCÁSTICO

con lo cual $c(t)$ permanece constante en dicho intervalo, $c(t) \equiv c_{0}$. De la misma forma, si en (22) se sustituye $\varepsilon_{0}$ por , se obtiene que también $c(t)$ permanece constante en el intervalo $\quad, c(t) \equiv c_{1}$. En consecuencia, de la ecuación (11) se tiene que

$$
A A: \quad c_{0}=\left\{\lambda\left[1+\alpha\left(\varepsilon_{0}+r\right)\right]\right\}^{\frac{1}{\gamma}}=c_{1}\left\{\lambda\left[1+\alpha\left(\varepsilon_{1}+r\right)\right]\right\}^{\frac{1}{\gamma}} .
$$

Esta ecuación expresa que la utilidad marginal del último peso gastado en $c_{0}$ es la misma que si se hubiera gastado en $c_{1}$. También observe que la tasa marginal de sustitución entre $c_{0}$ y $c_{1}$ permanece constante. Observe que en el plano $\left(c_{0}, c_{1}\right)\left(c_{0}\right.$ en el eje horizontal y $c_{1}$ en el vertical), el lugar geométrico $A A$ tiene pendiente positiva menor que uno. Ahora bien, de la restricción para la economía, dada en (20), se tiene que

$$
\left.\stackrel{\left(g_{1}, \infty\right)}{1>\frac{g \varepsilon_{1}}{\partial c_{0}}}\right|_{A A}=\left\{\begin{array}{l}
\left.\frac{1+\alpha\left(\varepsilon_{0}+r\right)}{1+\alpha\left(\varepsilon_{1}+r\right)}\right\}^{\frac{1}{\gamma}}>0 . \\
\text { con lo cual se define en el plano }\left(c_{0} c_{1}\right) \text { el lugar geométrico }
\end{array} \quad B(0)+\frac{y}{r}=\int_{0}^{T} c_{0} e^{-r t} \mathrm{~d} t+\int_{T}^{\infty} c_{1} e^{-r t} \mathrm{~d} t,\right.
$$

$$
B B: c_{1}=\left[y(t)+r B(0)-c_{0}\right] e^{r T}+c_{0} .
$$

Observe que

$$
\left.\frac{\partial c_{1}}{\partial c_{0}}\right|_{B B}=1-e^{r T}<0
$$

Es importante notar que, a lo largo de $A A, c_{0}>c_{1}$, ya que $\varepsilon_{0}<\varepsilon_{1}$. Note también que 
Evidentemente la intersección de $B B$ con la recta a $45^{\circ}$ proporciona el producto nacional bruto, $P N B_{0}$, al tiempo $t=0$. Dicha intersección se alcanza en

$$
c_{0}=y+r B(0)=G N P_{0} \text {. }
$$

Observe que si la tasa de devaluación fuera, para siempre, constante, es decir, si $T=0$, entonces

$$
c_{0}=c_{1}=y+r B(0)
$$

ya que lo anterior se sigue si en (25) se toma $c_{0}=c_{1}$. Sin embargo, si se espera que la política monetaria fuera temporal, es decir, si $0<T<\infty$, entonces

$$
0>c_{0}-c_{1}=\left[y-c_{0}+r B(0)\right] e^{r T},
$$

ya que la intersección de $A A \operatorname{con} B B$, es decir, el equilibrio, conduce a $c_{0}>c_{1}$, con lo cual se genera un déficit en cuenta corriente en el periodo de transición $[0, T]$. Después de $T$ la cuenta corriente se va a balancear y el nivel de consumo disminuye. Este nivel es menor que en el caso en que se espera que la política monetaria fuera permanente (i.e., cuando $[0, \infty)$ ).

\subsection{LONGITUD DEL PERIODO DE ESTABILIZACIÓN Y SU EFECTO SOBRE LA CUENTA CORRIENTE}

Una vez desarrollado el modelo básico, y después de haber obtenido algunos resultados preliminares, toca su turno al análisis de la longitud del periodo en que se aplica el programa de estabilización.

Es importante tener en mente que, la cuenta corriente refleja decisiones intertemporales de ahorro y desahorro de la economía. En la sección anterior el equilibrio se obtenía vía la intersección de las siguientes curvas: 
ANCLAS NOMINALES Y SU IMPACTO EN ECONOMÍAS PEQUEÑAS: UN ANÁLISIS COMPARATIVO ENTRE LOS ESQUEMAS DETERMINISTA Y ESTOCÁSTICO

$$
\begin{cases}A A: & c_{0}=\left\{\lambda\left[1+\alpha\left(\varepsilon_{0}+r\right)\right]\right\}^{\frac{1}{\gamma}}=c_{1}\left\{\lambda\left[1+\alpha\left(\varepsilon_{1}+r\right)\right]\right]^{\frac{1}{\gamma}} \\ B B: & c_{1}=\left[y(t)+r B(0)-c_{0}\right] e^{r T}+c_{0}\end{cases}
$$

Después de derivar parcialmente la ecuación para $B B$, con respecto de $T$, se encuentra que

$$
\frac{\partial c_{1}}{\partial T}=r\left[y+r B(0)-c_{0}\right] e^{r T}<0
$$

Consecuentemente, entre menor sea $T$ más plana será $B B$ (rotando sobre su intersección con la recta de $45^{\circ}$ ). También, observe que $A A$ no depende de $T$. Por lo tanto, al disminuir $T, c_{1}$ aumenta. De esta manera, entre más pequeña sea la longitud del periodo en que se aplica el programa de estabilización, mayor será el déficit en cuenta corriente en el periodo $[T, \infty)$. Por supuesto $c_{0}$ también aumenta. De lo anterior, se puede inferir que la no-neutralidad del dinero en el $\varepsilon(t) \quad$ inicio del periodo de estabilización podría hacerse más pronunciada.

Una política con $\varepsilon_{0}<\varepsilon_{1}$ y con $\mathrm{T}>0$ no conduce a un óptimo de Pareto, ya que hay fluctuaciones en $c(t)$. Un óptimo de Pareto se obtendría cuando $c(t)$ es constante e igual a $y+r B(0)=P N B_{0}$. En efecto, si un planeador central plantea el siguiente problema:

$$
\begin{gathered}
\text { Maximizar } \quad \int_{0}^{\infty} u[c(t)] e^{-r t} \mathrm{~d} t, \\
\text { sujeto a: } \int_{0}^{\infty}[c(t)-r B(0)-y] e^{-r t} \mathrm{~d} t,
\end{gathered}
$$

entonces la solución es

$$
c(t)=\text { constante }=y+r B(0)=G N P_{0}
$$

y, en consecuencia, una política de la forma $\varepsilon_{0}<\varepsilon_{1}$ y con $T>0$ no proporciona un óptimo de Pareto, ya que dicha política produce fluctuaciones en $c(t)$. Por último, otro resultado importante es que cualquier valor constante de

es 
preferible a que haya variaciones. Esto dramatiza la posibilidad de que un programa temporal de estabilización podría ser peor que hacer nada.

\section{MODIFICACIONES A LOS SUPUESTOS DEL PRIMER MODELO}

En el transcurso de esta sección se harán algunas modificaciones de considerable importancia a los supuestos del modelo hasta ahora desarrollado. Estas extensiones hacen que el modelado sea mucho más realista.

\subsection{BIENES NO-COMERCIABLES}

La forma más simple de introducir bienes no-comerciables es suponer que:

i. $\quad$ El individuo sólo consume bienes no-comerciables (bienes domésticos), $c_{n}(t)$,

ii. $\quad$ los bienes no-comerciables, $c_{n}(t)$, son producidos con bienes $c_{n}(t)=F\left[c_{s}(t)\right]$ comerciables $c_{S}(t)$, con función de producción la cual presenta rendimientos marginales decrecientes,

iii. la oferta de trabajo es perfectamente inelástica.

También, para ser más específicos se supondrá que

$$
F\left[c_{s}(t)\right]=\frac{\left[c_{s}(t)\right]^{1-\theta}}{1-\theta}, \quad 0<\theta<1
$$

Consecuentemente, el precio relativo $P(t)$ de $c_{n}(t)$ con respecto a $c_{s}(t)$, en equilibrio competitivo, se obtiene al resolver el problema

$$
\text { Maximizar } \Pi\left[c_{s}(t)\right]=p_{n}(t) \frac{\left[c_{s}(t)\right]^{1-\theta}}{1-\theta}-p_{s}(t) c_{s}(t)
$$


ANCLAS NOMINALES Y SU IMPACTO EN ECONOMÍAS PEQUEÑAS: UN ANÁLISIS COMPARATIVO ENTRE LOS ESQUEMAS DETERMINISTA Y ESTOCÁSTICO

De la condición de primer orden del problema de maximización se obtiene que

$$
P(t)=\frac{p_{n}(t)}{p_{s}(t)}=\left[c_{s}(t)\right]^{\theta}
$$

En consecuencia, el tipo de cambio real está dado por

$$
[P(t)]^{-1}=\left[c_{s}(t)\right]^{-\theta}
$$

Ahora bien, los saldos monetarios reales satisfacen

$$
m(t)=\frac{M(t)}{p_{s}(t)}
$$

donde $M(t)$ es el acervo de dinero doméstico. Por lo tanto, la restricción cashin-advance se escribe ahora como

$$
m(t) \geq \alpha P(t) c_{s}(t)
$$

De esta manera el individuo representativo tiene que resolver:

$$
\begin{gathered}
\text { Maximizar } \int_{0}^{\infty} u\left[c_{n}(t)\right] e^{-r t} \mathrm{~d} t, \\
\text { sujeto a: }\left\{\begin{array}{l}
a(0)+\frac{y}{r}+\int_{0}^{\infty} g(t) e^{-r t} \mathrm{~d} t=\int_{0}^{\infty}\left[c_{n}(t)+i(t) m(t)\right] e^{-r t} \mathrm{~d} t, \\
m(t)=\alpha P(t) c_{n}(t) .
\end{array}\right.
\end{gathered}
$$

Equivalentemente, en términos de bienes comerciables, 


$$
\begin{gathered}
\text { Maximizar } \int_{0}^{\infty} \frac{1}{1-\gamma}\left[\frac{\left[c_{s}(t)\right]^{1-\theta}}{1-\theta}\right]^{1-\gamma} e^{-r t} \mathrm{~d} t, \\
\text { sujeto a : }\left\{\begin{array}{l}
r a(t)-\dot{a}(t)+y+g(t)=c(t)-i(t) m(t), a_{0} \text { dado, } \\
m(t)=\alpha P(t) \frac{\left[c_{s}(t)\right]^{1-\theta}}{1-\theta} .
\end{array}\right.
\end{gathered}
$$

El Lagrangiano para este problema es

$$
L\left[c_{s}(t), \lambda\right]=\left\{\frac{1}{1-\gamma}\left[\frac{\left[c_{s}(t)\right]^{1-\theta}}{1-\theta}\right]^{1-\gamma}+\lambda\left\{y+g(t)-\frac{\left[c_{s}^{1-\theta}(t)\right]}{1-\theta}(1+P(t) \alpha i(t))\right\}\right\} e^{-r t}
$$

La condición de primer orden para una solución interior está dada por

$$
\lambda(1-\theta)^{-\gamma}\{(1+\alpha i(t)) P(t)\}=\left[c_{s}(t)\right]^{-\gamma(1-\theta)} .
$$

Observe que, cuando $\theta=0$ se obtiene (11). En equilibrio competitivo la condición anterior se transforma en

Si se considera de nuevo una política de la forma

$$
\varepsilon_{0}= \begin{cases}\varepsilon_{0}, & \text { para } 0 \leq t \leq T \\ \varepsilon_{1}, & \text { para } t>T\end{cases}
$$

$\operatorname{con} \varepsilon_{0}<\varepsilon_{1}$, se tendrá que 
ANCLAS NOMINALES Y SU IMPACTO EN ECONOMÍAS PEQUEÑAS: UN ANÁLISIS COMPARATIVO ENTRE LOS ESQUEMAS DETERMINISTA Y ESTOCÁSTICO

$$
c_{s}(t)= \begin{cases}c_{s 0}, & \text { para } t \in[0, T], \\ c_{s 1}, & \text { para } t \in(T, \infty) .\end{cases}
$$

Después de utilizar (33), se sigue que

$$
A A: \quad c_{s 0}=\left\{\left[1+\alpha\left(\varepsilon_{0}+r\right)\right] c_{s 0}^{\theta}\right\}^{\frac{1}{\theta}}=c_{s 1}\left\{\left[1+\alpha\left(\varepsilon_{1}+r\right)\right] c_{s 1}^{\theta}\right\}^{\frac{1}{\phi}},
$$

donde $\phi=\gamma(1-\theta)$. Al igual que antes, se tiene que

$$
B B: \quad c_{s 1}^{1-\theta}=(1-\theta)\left[y(t)+r B(0)-c_{s 0}^{1-\theta}\right] e^{r T}+c_{s 0}^{1-\theta} .
$$

Por lo tanto, al disminuir $T$ se tiene que en el nuevo equilibrio $c_{s 0}$ y $c_{s 1}$ serán mayores. En consecuencia, los precios relativos, $P(t)=\left[c_{s}(t)\right]^{\theta}$, aumentan en $[0, T] \mathrm{y}$, por lo tanto, el tipo de cambio real se está apreciando en $[0, T]$. Entre más pequeño es $T$ mayor es la apreciación del tipo de cambio real. Se puede decir algo más, después de $T$ el tipo de cambio real se va a depreciar por abajo del nivel, más que si $\mathcal{E}(t)$ hubiera permanecido constante en $[0, \infty)$.

\subsection{EL DINERO PROPORCIONA UTILIDAD POR SUS SERVICIOS DE LIQUIDEZ, Y DINERO Y CONSUMO SON SUSTITUTOS A LA EDGEWORTH}

La condición cash-in-advance es indudablemente muy restrictiva. Para relajar este supuesto se supondrá que la función de utilidad incluye saldos monetarios reales, reflejando con esto que el dinero proporciona utilidad al individuo por sus servicios de liquidez. En esta sección se considera el siguiente supuesto: la función de utilidad satisface $u_{c m}>0$, es decir, dinero y consumo son sustitutos a la Edgeworth. En este caso, la utilidad total descontada al tiempo $t=0$ está dada por 


$$
V(0)=\int_{0}^{\infty} u[c(t), m(t)] e^{-\rho t} \mathrm{~d} t
$$

en donde $u(\cdot, \cdot)$ es una función creciente en ambos argumentos, estrictamente cóncava con segundas derivadas parciales continuas que satisface

$$
\begin{array}{ll}
\lim _{c \rightarrow \infty} u_{c}(c, m)=0, \text { para } m \text { fija } & \lim _{m \rightarrow 0} u_{m}(c, m)=\infty, \text { para } c \text { fija, } \\
\lim _{c \rightarrow 0} u_{c}(c, m)=\infty, \text { para } m \text { fija } & \lim _{m \rightarrow \infty} u_{m}(c, m)=0, \text { para } c \text { fija. }
\end{array}
$$

Se supone ahora que la función de utilidad $u$ tiene la siguiente forma funcional. Sea $0<\alpha<1$,

En este caso, $u_{m m}<0, u_{c c}<0$ y $u_{c m} \geq 0$. El Hamiltoniano ${ }^{2}$ está dado por

$$
H[c(t), m(t), \lambda(t)]=u[c(t), m(t)]+\lambda(t)\{y+g(t)-c(t)-i(t) m(t)\}
$$

Las condiciones de primer orden para una solución interior son:

$$
H_{c}=0, \quad H_{m}=0, \quad \mathrm{y} \quad-H_{a}=\lambda(t)-r \lambda(t)
$$

de donde se tiene que $\lambda(t)=0$, con lo cual $\lambda(t)$ es constante, . También se obtiene que, para toda $R>1$ la condición necesaria de óptimo es

\footnotetext{
2 Ver apéndice a esta sección.
} 
ANCLAS NOMINALES Y SU IMPACTO EN ECONOMÍAS PEQUEÑAS: UN ANÁLISIS COMPARATIVO ENTRE LOS ESQUEMAS DETERMINISTA Y ESTOCÁSTICO

$$
\left\{\begin{array}{l}
\frac{\alpha\left(c(t)^{\alpha} m(t)^{1-\alpha}\right)^{-R}}{c(t)^{1-\alpha}}=\lambda, \\
\frac{(1-\alpha)\left(c(t)^{\alpha} m(t)^{1-\alpha}\right)^{-R}}{m(t)^{\alpha}}=\lambda i(t),
\end{array}\right.
$$

de donde

$$
m(t)=\left(\frac{(1-\alpha)}{\alpha i(t)}\right)^{1 / \alpha} c(t)^{(1-\alpha) / \alpha} .
$$

Si se sustituye (40) en la primera ecuación de (39), se encuentra que para toda $R>0$

$$
c(t)=\left(\frac{\lambda}{\alpha}\left(\frac{(1-\alpha)}{\alpha i(t)}\right)^{\Lambda}\right)^{\frac{1}{\Psi}},
$$

donde

$$
\Lambda=\frac{(1-\alpha) R}{\alpha} \quad \text { y } \quad \Psi=\alpha(1-R)-\frac{(1-\alpha)^{2}}{\alpha}-1
$$

Si de nuevo se vuelve a tomar una política de la forma:

$$
\varepsilon_{0}= \begin{cases}\varepsilon_{0}, & \text { para } 0 \leq t \leq T, \\ \varepsilon_{1}, & \text { para } t>T,\end{cases}
$$

$\operatorname{con} \varepsilon_{0}<\varepsilon_{1}$, se sigue 
e inmediatamente se obtiene que, en el equilibrio $c_{0}>c_{1}$. También se puede proceder considerando, en forma más general, una función de utilidad $u=u(c, m)$ con $u_{m m}<0, u_{c c}<0 \mathrm{y} \quad$, de tal forma que (39) se reescriba como:

$$
\frac{u_{m}(c(t), m(t))}{u_{c}(c(t), m(t))}=i(t)
$$

lo cual significa que, la tasa marginal de sustitución entre saldos monetarios reales y consumo es igual al costo de oportunidad de mantener dichos saldos. Si se define ahora

$$
G[c(t), m(t), i(t)]=u_{m}[c(t), m(t)]-i(t) u_{c}[c(t), m(t)]=0,
$$

entonces

$$
1 A_{A}:>\varepsilon_{0}=\left(\varepsilon_{0}+r\right)^{\Lambda /}
$$

$$
G_{m}=u_{m m}-i u_{m c}<0
$$

con lo cual se está en posición de aplicar el teorema de la función implícita a $G$. Por lo tanto existe, al menos localmente, una función $L$ tal que

$$
m(t)=L[c(t), i(t)]
$$

Esta función satisface

$$
L_{c}=\frac{\partial m}{\partial c}=-\frac{G_{c}}{G_{m}}>0
$$

y

$$
L_{i}=\frac{\partial m}{\partial i}=-\frac{G_{i}}{G_{m}}<0,
$$


ANCLAS NOMINALES Y SU IMPACTO EN ECONOMÍAS PEQUEÑAS: UN ANÁLISIS COMPARATIVO ENTRE LOS ESQUEMAS DETERMINISTA Y ESTOCÁSTICO

ya que

$$
G_{m}=u_{m m}-i u_{m c}<0, \quad G_{c}=u_{m c}-i u_{c c}>0, \quad G_{i}=-u_{c}<0 .
$$

Por lo tanto, se puede reescribir una de las condiciones de primer orden como

$$
u_{c}=[c(t), L[c(t), i(t)]]=\lambda,
$$

y como $i(t)=r(t)+\varepsilon(t)$, entonces (44) se puede expresar de la siguiente forma

Si se vuelve a tomar una política de la forma (41), se obtiene que

$$
A A: f[c(t), \varepsilon(t)]=u_{c}=[c(t), L[c(t), r+\varepsilon(t)]]_{\bar{c}(t)}=\left\{\begin{array}{lll}
c_{0}, & \text { para } & t \in[0, T], \\
c_{1}, & \text { para } & t \in(T, \infty)
\end{array}\right.
$$

Observe ahora que, a partir de (45) se obtiene

$$
0>f[c(t), \varepsilon(t)]=u_{c c}=\left[c(t), L[c(t), r+\varepsilon(t)]=\frac{\partial \lambda}{\partial c} .\right.
$$

De nuevo esta condición nos habilita para usar el teorema de la función implícita, con lo cual

$$
\frac{\partial c}{\partial \varepsilon}=-\frac{f_{\varepsilon}}{f_{c}}=-u_{c m}
$$

Por lo tanto se tiene una curva como la de $A A$, y como 
Francisco Venegas Martínez / Abigail Rodríguez Nava

$$
B B: c_{1}=\left[y(t)+r B(0)-c_{0}\right] e^{r T}+c_{0},
$$

sigue siendo válida, todas las conclusiones anteriores se mantienen.

\subsection{EL DINERO Y EL CONSUMO NO SON PERFECTOS SUSTITUTOS}

Observe que si $u_{c m}<0$, entonces la curva $A A$ estará por arriba de la línea de $45^{\circ}$, con lo cual $c_{0}<c_{1}$, es decir, ahora la economía experimenta un superávit en cuenta corriente en el periodo de transición $[0, T]$. Finalmente, note que si $u_{c m}=0$, entonces no hay efectos reales en la economía.

\subsection{NO HAY MOVILIDAD DE CAPITAL.}

En este caso los resultados serían drásticamente diferentes. Por ejemplo, si ni el gobierno, ni el sector privado tuvieran bonos internacionales, es decir, $B(0)=b(0)=F(0)$, la única solución posible sería $c=y$ en todo momento, independientemente de la política cambiaria que se adopte.

\section{EL MODELO ESTOCÁSTICO}

Como antes, con el propósito de obtener soluciones analíticas en un modelo estocástico, se mantendrá la estructura de la economía tan simple como sea posible. Es importante mencionar que otros modelos, sobre el tema en cuestión, disponibles en la literatura en el marco estocástico son Uribe (2002) y Uribe y Mendoza (2000).

\subsection{DINÁMICA DEL TIPO DE CAMBIO Y PRECIOS}

Considere una economía pequeña y abierta con agentes idénticos con vida infinita. La economía produce y consume un solo bien. Se supone que el bien 
ANCLAS NOMINALES Y SU IMPACTO EN ECONOMÍAS PEQUEÑAS: UN ANÁLISIS COMPARATIVO ENTRE LOS ESQUEMAS DETERMINISTA Y ESTOCÁSTICO

es comerciable internacionalmente y que el nivel general de precios en la economía doméstica, $P_{t}$, es determinado por la condición de poder de paridad de compra, a saber, $P_{t}=P_{t}^{*} e_{t}$, donde $P_{t}^{*}$ es el precio en moneda extranjera del bien en el resto del mundo y $e_{t}$ es el tipo de cambio nominal. Se supone, por simplicidad, que $P_{t}^{*}$ es igual a 1 y que el valor inicial del tipo de cambio, $e_{0}$, es conocido y también igual a 1.

Se supone que el número de devaluaciones esperadas, i.e. los saltos en el tipo de cambio, por unidad de tiempo, siguen un proceso de Poisson $N_{t}$ con intensidad $\lambda$, de tal manera que

mientras que

$$
\mathrm{P}^{(N)}\{\text { ningún salto en } \mathrm{d} t\}=\mathrm{P}^{(N)}\left\{\mathrm{d} N_{t}=0\right\}=1-\lambda \mathrm{d} t+o(\mathrm{~d} t) .
$$

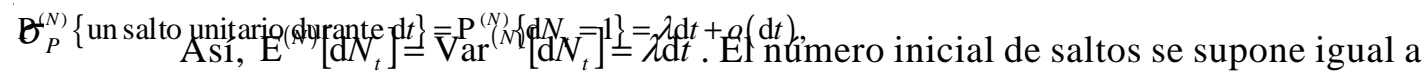
cero, es decir, $N_{0}=0$.

Considere un proceso de Wiener $\left(Z_{t}\right)_{t \geq 0}$ definido en un espacio de probabilidad fijo con su filtración aumentada $\left(\Omega^{(Z)}, F^{(Z)},\left(F_{t}^{(Z)}\right)_{t \geq 0}, \mathrm{P}^{(Z)}\right)$. Se supone que el consumidor percibe que la tasa de inflación esperada, $\mathrm{d} P_{t} / P_{t} \mathrm{y}$, por lo tanto, la tasa esperada de devaluación, $\mathrm{d} e_{t} / e_{t}$, sigue un movimiento geométrico Browniano con saltos de Poisson definido por la siguiente ecuación diferencial estocástica:

$$
\frac{\mathrm{d} P_{t}}{P_{t}}=\frac{\mathrm{d} e_{t}}{e_{t}}=\varepsilon \mathrm{d} t+\sigma_{P} \mathrm{~d} Z_{t}+\gamma \mathrm{d} N_{t}
$$

donde $\varepsilon$ es la tasa media esperada de devaluación, condicionada a que no se presenten saltos, es la volatilidad instantánea del nivel general de precios, 
y es el tamaño medio esperado de un salto en el tipo de cambio. El proceso $Z_{t}$ se supone independiente de $N_{t}$. En lo que se sigue, , , y son constantes positivas.

\subsection{SALDOS MONETARIOS REALES}

El agente mantiene saldos monetarios reales, donde $M_{t}$ es el acervo nominal de dinero. La tasa de rendimiento estocástica por la tenencia de saldos reales, $\mathrm{d} R_{m}$, está dada por el cambio porcentual de los saldos reales. Al aplicar el lema de Itô para procesos de difusión de saltos al inverso del nivel de precios, con (49) como el proceso subyacente (véase el apéndice a la sección 5), se obtiene:

$$
\mathrm{d} R_{m}=\mathrm{d}\left(\frac{M_{t}}{P_{t}}\right) /\left(\frac{M_{t}}{P_{t}}\right)=\left(-\varepsilon+\sigma_{P}^{2}\right) \mathrm{d} t-\sigma_{P} \mathrm{~d} Z_{t}-\left(\frac{\gamma}{1+\gamma}\right) \mathrm{d} N_{t} .
$$

\subsection{BONOS INTERNACIONALES}

El agente también tiene acceso a un bono internacional, $b_{t}$, que paga una tasa de interés real libre de riesgo, $r$, que es constante para todos los plazos. En este caso, se satisface

$$
\mathrm{d} b_{t}=r b_{t} \mathrm{~d} t, \quad b_{0} \text { dado. }
$$

Es decir, el bono paga $r$ unidades del bien de consumo por unidad de tiempo. Note que, por el supuesto de economía pequeña, el agente toma $r$ como dada.

\subsection{UNA ECONOMÍA DEL TIPO CASH-IN-ADVANCE}

Considere una restricción del tipo cash-in-advance de la forma Clower-LucasFeenstra: 
ANCLAS NOMINALES Y SU IMPACTO EN ECONOMÍAS PEQUEÑAS: UN ANÁLISIS COMPARATIVO ENTRE LOS ESQUEMAS DETERMINISTA Y ESTOCÁSTICO

$$
m_{t}=\alpha c_{t}
$$

donde $c_{t}$ es el consumo y $\alpha>0$ es el tiempo que se mantiene el dinero para financiar el consumo. La condición (52) es crítica para ligar la política cambiaria y el consumo. De esta forma, la devaluación actúa como un impuesto estocástico en los saldos monetarios reales.

\subsection{INGRESO LABORAL}

El consumidor representativo administra y trabaja en su propio negocio. Se supone que el ingreso laboral, $y_{t}$, es transformado en activos reales, , a una tasa incierta $v_{t}$, de tal manera que

$$
y_{t}=v_{t} a_{t}
$$

y $v_{t}$ es conducido por un movimiento geométrico Browniano. Sea $\left(U_{t}\right)_{t \geq 0}$ un

$a_{t}$ proceso de Wiener (movimiento Browniano) definido en un espacio de probabilidad fijo equipado con su filtración aumentada $\left(\Omega^{(U)}, F^{(U)},\left(F_{t}^{(U)}\right)_{t \geq 0}, \mathrm{P}^{(U)}\right)$. Se supone que la tasa de variación del ingreso, $v_{t}$, está dada por

$$
\frac{\mathrm{d} v_{t}}{v_{t}}=\bar{v} \mathrm{~d} t+\sigma_{v} \mathrm{~d} \tilde{Z}_{t}, \quad y_{0}>0
$$

donde

$$
\tilde{Z}_{t}=\rho Z_{t}+\sqrt{1-\rho 2} U_{t}
$$

$\mathrm{y}$

$$
\operatorname{Cov}\left(\mathrm{d} Z_{t}, \mathrm{~d}\left(\rho Z_{t}+\sqrt{1-\rho 2} U_{t}\right)\right)=\rho \mathrm{d} t
$$


$\bar{v}$ y $\sigma_{v}$ son constantes positivas, $\mathrm{y}$

es la correlación entre cambios en la inflación y cambios en el ingreso laboral. Los procesos $N_{t}, Z_{t} \mathrm{y}$ $U_{t}$ se suponen independientes dos a dos.

\subsection{PROBLEMA DE DECISIÓN DEL CONSUMIDOR}

En esta sección se caracterizarán las decisiones óptimas de consumo y portafolio del agente representativo. Para ello se obtendrán soluciones explícitas de dichas decisiones, lo cual hará más sencilla la comprensión de los aspectos relevantes de los programas temporales de estabilización. La acumulación de la riqueza del consumidor en términos del portafolio $\quad, 1-w_{t}=b_{t} / a_{t}, \mathrm{y}$ del consumo, $c_{t}$, está dada por el siguiente sistema de ecuaciones diferenciales estocásticas:

$$
\begin{aligned}
& \mathrm{d} a_{t}=a_{t} w_{t} \mathrm{~d} R_{m}+a_{t}\left(1-w_{t}\right) \mathrm{d} R_{b}+\left(v_{t} a_{t}-c_{t}\right) \mathrm{d} t, \quad a_{0}=m_{0}+b_{0}>0, \\
& \mathrm{~d} v_{t}=\bar{v} v_{t} \mathrm{~d} t+\sigma_{v} v_{t}\left(\rho \mathrm{d} Z_{t}+\sqrt{1-\rho 2} \mathrm{~d} U_{t}\right), \quad v_{0}>0,
\end{aligned}
$$

donde $\mathrm{d} R_{b}=\mathrm{d} b_{t} / b_{t}$. Si se sustituyen las ecuaciones (50), (51) y (52) en la pri$\boldsymbol{\omega}_{t}=e n_{t} /\left(t_{t} 1,1\right)$ mera ecuación del sistema de ecuaciones diferenciales (56), se tiene que

$$
\mathrm{d} a_{t}=a_{t}\left[\left(r-\mu w_{t}+v_{t}\right) \mathrm{d} t-w_{t} \sigma_{P} \mathrm{~d} Z_{t}-w_{t}\left(\frac{\gamma}{1-\gamma}\right) \mathrm{d} N_{t}\right],
$$

donde $\mu=\alpha^{-1}+r+\varepsilon-\sigma^{2}$.

\section{7 ÍNDICE DE SATISFACCIÓN}

La utilidad total esperada del tipo de von Neumann-Morgenstern al tiempo $t, V_{t}$, de un consumidor (representativo) competitivo adverso al riesgo se supone de la forma:

$$
V_{t}=E\left\{\int_{t}^{\infty} \log \left(c_{s}\right) e^{-r s} \mathrm{~d} s \mid F_{t}\right\}
$$


ANCLAS NOMINALES Y SU IMPACTO EN ECONOMÍAS PEQUEÑAS: UN ANÁLISIS COMPARATIVO ENTRE LOS ESQUEMAS DETERMINISTA Y ESTOCÁSTICO

donde $F_{t}=F_{t}^{(Z)} \otimes F_{t}^{(U)}$ representa la información total disponible al tiempo $t$. Se emplea la función de utilidad logarítmica con el propósito de obtener soluciones cerradas que hagan el análisis más sencillo. Observe también que la tasa subjetiva de descuento del agente ha sido igualada a la tasa de interés $r$ para evitar dificultades técnicas innecesarias.

\subsection{LA ECUACIÓN DE HAMILTON-JACOBI-BELLMAN}

La ecuación de Hamilton-Jacobi-Bellman para el problema de control óptimo estocástico, en el que se maximiza la utilidad esperada del agente, sujeto a su restricción presupuestal intertemporal, es:

$$
\begin{aligned}
& \lambda I\left(a_{t}, v_{t}, t\right)-I_{t}\left(a_{t}, v_{t}, t\right)-I_{v}\left(a_{t}, v_{t}, t\right) \bar{v} v_{t}-\frac{1}{2} I_{v v}\left(a_{t}, v_{t}, t\right) v_{t}^{2} \sigma_{v}^{2}-I_{a}\left(a_{t}, v_{t}, t\right) a_{t}\left(r+v_{t}\right) \\
= & \max _{w}\left\{\log \left(\alpha^{-1} a_{t} w_{t}\right) e^{-r t}-I_{a}\left(a_{t}, v_{t}, t\right) a_{t} \mu w_{t}+\frac{1}{2} I_{a a}\left(a_{t}, v_{t}, t\right) a_{t}^{2} w_{t}^{2} \sigma_{P}^{2}\right. \\
& \left.-I_{a v}\left(a_{t}, v_{t}, t\right) a_{t} v_{t} w_{t} \sigma_{P} \sigma_{v} \rho+\lambda I\left(a_{t}\left(\frac{1+\gamma\left(1-w_{t}\right)}{1+\gamma}\right), v_{t}, t\right)\right\},
\end{aligned}
$$

donde

$$
I\left(a_{t}, v_{t}, t\right)=\max _{w} E_{t}\left\{\int_{t}^{\infty} \log \left(\alpha^{-1} a_{s} w_{s}\right) e^{-r s} \mathrm{~d} s \mid F_{t}\right\}
$$

es la función de utilidad indirecta (o función del bienestar económico) o función de valor del consumidor, e $I_{a}\left(a_{t}, v_{t}, t\right)$ es la variable de coestado.

\subsection{REDUCCIÓN DE LA DIMENSIÓN DEL PROBLEMA}

Dado el factor de descuento exponencial en la ecuación (60), se define $I_{a}\left(a_{t}, v_{t}, t\right)$ en forma separable en el tiempo como:

$$
I\left(a_{t}, v_{t}, t\right) \equiv F\left(a_{t}, v_{t}\right) e^{-r t}
$$


Por lo tanto, la ecuación (60) se transforma en

$$
\begin{aligned}
& (\lambda+r) F\left(a_{t}, v_{t}\right)-F_{v}\left(a_{t}, v_{t}\right) \bar{v} v_{t}-\frac{1}{2} F_{v v}\left(a_{t}, v_{t}\right) v_{t}^{2} \sigma_{v}^{2}-F_{a}\left(a_{t}, v_{t}\right) a_{t}\left(r+v_{t}\right) \\
= & \max _{w}\left\{\log \left(\alpha^{-1} a_{t} w_{t}\right)-F_{a}\left(a_{t}, v_{t}\right) a_{t} \mu w_{t}+\frac{1}{2} F_{a a}\left(a_{t}, v_{t}\right) a_{t}^{2} w_{t}^{2} \sigma_{P}^{2}\right. \\
& \left.-F_{a v}\left(a_{t}, v_{t}\right) a_{t} v_{t} w_{t} \sigma_{P} \sigma_{v} \rho+\lambda F\left(a_{t}\left(\frac{1+\gamma\left(1-w_{t}\right)}{1+\gamma}\right), v_{t}\right)\right\} .
\end{aligned}
$$

Se postula como posible candidato de solución de la ecuación diferencial ordinaria (61) a:

$$
F\left(a_{t}, v_{t}\right)=\theta_{0}+\theta_{1} \log \left(a_{t} v_{t}\right)+\phi\left(v_{t} ; \theta_{2}, \theta_{3}\right)
$$

donde $\theta_{0}, \theta_{1}$ y $\phi\left(v_{t}\right)$ son determinados a través de la ecuación (61). Si se sustituye la ecuación (62) en la ecuación (61), se tiene que

$$
\begin{aligned}
& r\left(\theta_{0}+\theta_{1} \log \left(a_{t}\right)\right)- \\
& +r \phi\left(v_{t}\right)-\phi^{\prime}\left(v_{t}\right) \bar{v} v_{t} \\
& =\max _{w}\left\{\operatorname { l o g } \left(\alpha^{-1} a_{t} w\right.\right. \\
& +\lambda \theta_{1} \log \left(\frac{1+\gamma(1-}{1+\gamma}\right.
\end{aligned}
$$

\subsection{CONDICIONES DE PRIMER ORDEN Y DETERMINACIÓN DE COEFICIENTES}

Después de calcular las condiciones de primer orden del problema de optimización intertemporal se obtiene que $w_{t} \equiv w$ es independiente del tiempo, así como la relación 
ANCLAS NOMINALES Y SU IMPACTO EN ECONOMÍAS PEQUEÑAS: UN ANÁLISIS COMPARATIVO ENTRE LOS ESQUEMAS DETERMINISTA Y ESTOCÁSTICO

$$
\frac{1}{\theta_{1} w}-\frac{\lambda \gamma}{1+\gamma(1-w)}=\mu+w \sigma_{P}^{2}
$$

Se selecciona $\phi\left(v_{t}\right)$ como solución de la ecuación diferencial de segundo orden dada por

$$
r \phi\left(v_{t}\right)-\phi^{\prime}\left(v_{t}\right) \bar{v} v_{t}-\frac{1}{2} \phi^{\prime \prime}\left(v_{t}\right) v_{t}^{2} \sigma_{v}^{2}+r \theta_{1} \log \left(v_{t}\right)-\theta_{1} v_{t}=0
$$

Los coeficientes $\theta_{0}$ y $\theta_{1}$ son determinados de la ecuación (61) al sustituir $w^{*}$ óptima. Así, $\theta_{1}=r^{-1}$, lo que hace que el coeficiente de $\log \left(a_{t}\right)$ en la ecuación (63) sea cero y, en consecuencia,

$$
\begin{aligned}
& \theta_{0}=\frac{1}{r} \log \left(\alpha^{-1} w^{*}\right) \\
& -\frac{1}{r^{2}}\left[\left(\alpha^{-1}+r+\varepsilon-\sigma_{P}^{2}\right) w^{*}+\frac{1}{2}\left(w^{*} \sigma_{P}\right)^{2}-\bar{v}-r+\frac{1}{2} \sigma_{P}^{2}-\lambda \log \left(\frac{1+\gamma\left(1-w^{*}\right)}{1+\gamma}\right)\right] .
\end{aligned}
$$

La utilidad logarítmica conduce a que $w$ dependa solamente de los parámetros que determinan las características estocásticas de la economía y, por lo tanto, $w$ es constante. Es decir, la actitud del consumidor hacia el riesgo cambiario es independiente de su riqueza, i.e. el nivel de riqueza resultante en cualquier instante no tiene relevancia para las decisiones de portafolio. Más aún, debido a la utilidad logarítmica, el coeficiente de correlación, $\rho \in(-1,1)$, no juega papel alguno en las decisiones del consumidor. Por último, es importante señalar que la ecuación (64) es cúbica, por lo que tiene al menos una raíz real. La solución de la ecuación (65) es

$$
\phi\left(v_{t}\right)-\theta_{2} v_{t}^{\lambda_{1}}+\theta_{3} v_{t}^{\lambda_{2}}-\frac{1}{\bar{v}} \log \left(v_{t}\right)\left(1+\frac{2}{\left(\sigma_{v}^{2}+2 \bar{v}\right)} v_{t}\right)+\frac{1}{\bar{v}}\left(\frac{\sigma_{v}^{2}}{2 \bar{v}}-1\right),
$$

donde 
Francisco Venegas Martínez / Abigail Rodríguez Nava

$$
\lambda_{1}=\frac{4 r}{\left(2 \bar{v}-\sigma_{v}^{2}\right)+\sqrt{\left(2 \bar{v}-\sigma_{v}^{2}\right)^{2}+8 r \sigma_{v}^{2}}}
$$

$\mathrm{y}$

$$
\lambda_{2}=\frac{4 r}{\left(2 \bar{v}-\sigma_{v}^{2}\right)-\sqrt{\left(2 \bar{v}-\sigma_{v}^{2}\right)^{2}+8 r \sigma_{v}^{2}}}
$$

Los coeficientes $\theta_{2}$ y son obtenidos de tal manera que $\phi\left(v_{0}\right)=0$ y La primera condición inicial asegura que el bienestar económico,

$$
W \equiv I\left(a_{0}, v_{0}, 0\right)=F\left(a_{0}, v_{0}\right)=\theta_{0}+\theta_{1} \log \left(a_{0}, v_{0}\right),
$$

sea independiente de la selección de $\phi$. La segunda condición, conduce a

$$
\left.\frac{\partial g}{\partial v}\right|_{v=v_{0}} \mid z_{0}\left(0=1 \emptyset \frac{1}{r v_{0}}>0\right.
$$

la cual asegura que un incremento en $v_{0}$ mejora el bienestar económico. Por supuesto, esta segunda condición también asegura una solución única, $\phi$, de la ecuación diferencial (65).

\subsection{UNA ASIGNACIÓN VIABLE DEL PORTAFOLIO}

La ecuación (64) es cúbica con una raíz negativa y dos raíces positivas. Esto puede ser visto al intersectar la línea recta definida por el lado derecho de la ecuación (64) con la gráfica definida por el lado izquierdo de (64). En este caso, hay solamente una intersección que proporciona un estado estacionario único de la proporción de la riqueza asignada al consumo 
ANCLAS NOMINALES Y SU IMPACTO EN ECONOMÍAS PEQUEÑAS: UN ANÁLISIS COMPARATIVO ENTRE LOS ESQUEMAS DETERMINISTA Y ESTOCÁSTICO

\subsection{EXPERIMENTOS DE POLÍTICA ECONÓMICA Y ESTÁTICA COMPARATIVA}

A continuación se obtiene un conjunto de resultados relevantes. En primer lugar, observe que un aumento permanente en la tasa de devaluación da como resultado un incremento en el costo de oportunidad futuro de comprar bienes, lo cual conduce, a su vez, a una disminución permanente de la proporción de la riqueza destinada al consumo futuro. Para ver esto se calcula la derivada de la ecuación (64) con respecto de $\varepsilon$, lo cual lleva a

donde

$$
\frac{\partial w^{*}}{\partial \varepsilon}=-\Delta^{-1}<0, \quad \Delta=\left[\frac{r}{\left(w^{*}\right)^{2}}+\frac{\lambda \gamma^{2}}{\left[1+\gamma\left(1-w^{*}\right)\right]^{2}}+\sigma_{P}^{2}\right]>0
$$

Es decir, un aumento en $\varepsilon$ conduce a una reducción en la tenencia futura de saldos reales y, por lo tanto, lleva a una disminución en el futuro consumo; tal y como lo predecía el modelo básico de la sección 2. Es necesario destacar que en el marco estocástico el consumo es una variable aleatoria, siendo la variable de decisión la proporción de la riqueza que el consumidor asigna a la tenencia de saldos reales y, de acuerdo a la condición cash-in-advance, estos últimos se utilizan para financiar el consumo.

\section{CONCLUSIONES}

Con base en un primer modelo, llamado el modelo básico, se ha mostrado que una política de estabilización basada en una disminución temporal de la tasa de 
devaluación genera un déficit temporal sobre la cuenta corriente en economías con un bien (comerciable), y una apreciación el tipo de cambio real en economías con dos bienes (comerciables y no comerciables). En el primer caso (un bien), entre más corto sea el periodo de estabilización, mayor será el déficit en la cuenta corriente. En el segundo caso (dos bienes), entre más corto sea el periodo de estabilización, mayor será la apreciación del tipo de cambio real.

Asimismo, se mostró que bajo la extensión se tiene una apreciación del tipo de cambio real. Entre más corto es el periodo de estabilización mayor será la apreciación del tipo de cambio real. Bajo la modificación (b) no se tienen cambios sustanciales en las conclusiones del primer modelo. Sin embargo, bajo las modificaciones $(c)$ y $(d)$, los resultados pueden ser completamente diferentes. Por ejemplo, con respecto de $(d)$, si ni el gobierno ni el sector privado tienen bonos internacionales, se tendría entonces un efecto de economía cerrada.

Es importante destacar que, los resultados del modelo estocástico de estabilización temporal son consistentes con los del modelo básico aunque, en el marco estocástico, cuando los agentes son expuestos al riesgo, se afectan de manera sensible su comportamiento y sus expectativas. Por ejemplo, si un consumidor-inversionista toma decisiones de consumo e inversión en un ambiente estocástico y tiene acceso a bonos y a un activo con riesgo (en este caso, saldos reales estocásticos), se tiene, infortunadamente, que la trayectoria de consumo no puede ser determinada porque el consumo se convierte en variable aleatoria, situación que está más acorde con la realidad. En consecuencia, la consideración del riesgo conlleva a cambios cualitativos y cuantitativos drásticos en las decisiones de consumo y portafolio de los agentes.

Por último, es importante mencionar que el hecho de combinar un movimiento Browniano con un proceso Poisson aporta nuevos elementos para llevar a cabo experimentos de simulación e investigación empírica sobre diversos hechos estilizados registrados en estabilizaciones temporales. 
ANCLAS NOMINALES Y SU IMPACTO EN ECONOMÍAS PEQUEÑAS: UN ANÁLISIS COMPARATIVO ENTRE LOS ESQUEMAS DETERMINISTA Y ESTOCÁSTICO

\section{BIBLIOGRAFÍA}

Calvo, G. A. y C. A. Végh (1999), "Inflation stabilization and balance-of-payments crises in developing countries", en: J. Taylor, W. Woodford eds., Handbook of Macroeconomics, Vol. 1C. North-Holland, Amsterdam, pp. 1531-1614.

Drazen, A. y E. Helpman (1988), "Stabilization with exchange rate management under uncertainty", en: E. Helpman, A. Razin y E. Sadka eds., Economic effects of the government budget (MIT Press, Cambridge, MA).

Dorfman, R. (1969), “An Economic Interpretation of Optimal Control Theory”, The American Economic Review, Vol. 59, No. 5, pp. 817-831.

Kamien, M. I. y N. L. Schwartz (1981), Dynamic Optimization, The Calculus of Variations and Optimal Control in Economics and Management, NorthHolland.

Uribe, M. (2002), "The price-consumption puzzle of currency pegs", Journal of Monetary Economics, Vol. 49, No. 3, pp. 533-569.

Uribe, M. y E. G. Mendoza (2000), "Devaluation risk and the business-cycle implications of exchange rate management". Carnegie-Rochester Conference Series on Public Policy, Vol. 53, No. 1, pp. 239-296.

Venegas-Martínez, F. (2001), "Temporary Stabilization: A Stochastic Analysis", Journal of Economic Dynamics and Control, Vol. 25, No. 9, pp. 14291449. 


\section{APÉNDICE A LA SECCIÓN 3}

El problema planteado de maximización de utilidad en la sección 3 puede ser resuelto utilizando cálculo de variaciones (Ecuaciones de Euler-Lagrange) o control óptimo (Principio del máximo de Pontryagin). (Como ejemplo véase Dorfman, 1969; Kamien y Schwartz, 1981).

Los siguientes planteamientos son equivalentes; algunos consideran Hamiltonianos descontados:
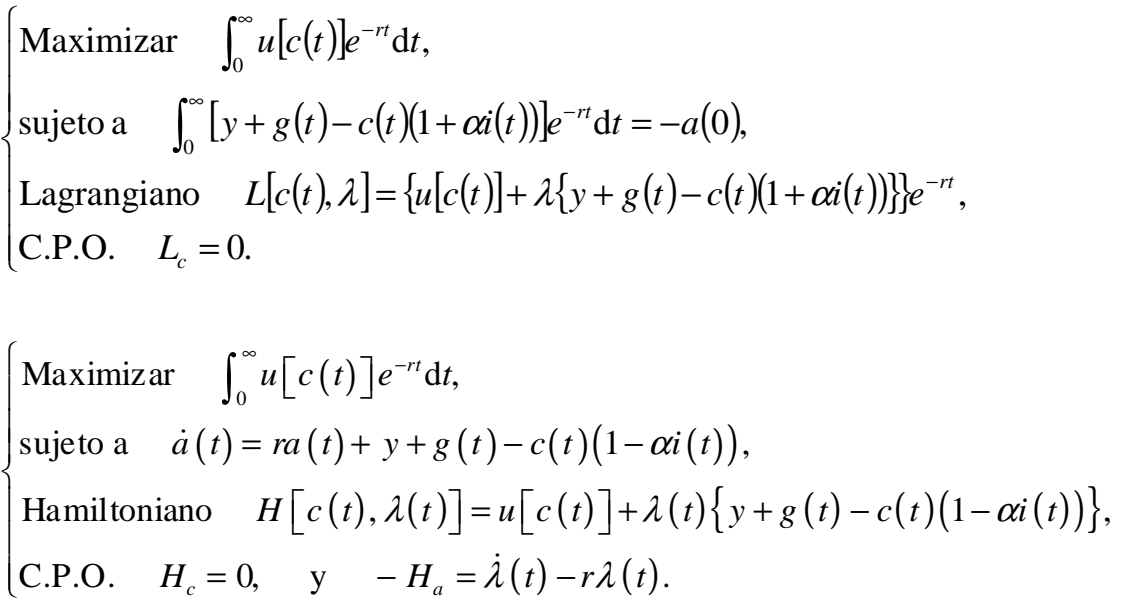

$\left\{\begin{array}{l}\text { Maximizar } \quad \int_{0}^{\infty} u[c(t)] e^{-r t} \mathrm{~d} t, \\ \text { sujeto a } \dot{a}(t)=r a(t)+y+g(t)-c(t)(1-\alpha i(t)), \\ \text { Hamiltoniano } H[c(t), \lambda(t)]=u[c(t)] e^{-r t}+\lambda(t) \dot{a}(t),\end{array}\right.$

C.P.O. $\quad H_{c}=0, \quad H_{\lambda}=\dot{a}(t), \quad$ y $\quad-H_{a}=\dot{\lambda}(t)$.




ANCLAS NOMINALES Y SU IMPACTO EN ECONOMÍAS PEQUEÑAS: UN ANÁLISIS COMPARATIVO ENTRE LOS ESQUEMAS DETERMINISTA Y ESTOCÁSTICO

\section{APÉNDICE A LA SECCIÓN 4}

El problema planteado de maximización de utilidad en la sección 4 puede ser resuelto utilizando cálculo de variaciones (Ecuaciones de Euler-Lagrange) o control óptimo (Principio de Pontryagin).

Los siguientes planteamientos son equivalentes:

$$
\begin{aligned}
& \left\{\begin{array}{l}
\text { Maximizar } \int_{0}^{\infty} u[c(t), m(t)] e^{-r t} \mathrm{~d} t, \\
\text { sujeto a } \quad \int_{0}^{\infty}[y+g(t)-c(t)(1+\alpha i(t))] e^{-r t} \mathrm{~d} t=-a(0), \\
\text { Lagrangiano } \quad L[c(t), m(t), \lambda]=\{u[c(t), m(t)]+\lambda\{y+g(t)-c(t)(1+\alpha i(t))\}\} e^{-r t}, \\
\text { C.P.O. } \quad L_{c}=0, \quad L_{m}=0 .
\end{array}\right. \\
& \left\{\begin{array}{l}
\text { Maximizar } \quad \int_{0}^{\infty} u[c(t), m(t)] e^{-r t} \mathrm{~d} t, \\
\text { sujeto a } \quad a(t)=r a(t)+y+g(t)-c(t)(1-\alpha i(t)), \\
\text { Hamiltoniano } \quad H[c(t), m(t), \lambda(t)]=u[c(t), m(t)]+\lambda(t)\{y+g(t)-c(t)(1-\alpha i(t))\} \\
\text { C.P.O. } \quad H_{c}=0, \quad H_{m}=0 \quad \mathrm{y} \quad-H_{a}=\lambda(t)-r \lambda(t) .
\end{array}\right. \\
& \left\{\begin{array}{l}
\text { Maximizar } \int_{0}^{\infty} u[c(t), m(t)] e^{-r t} \mathrm{~d} t, \\
\text { sujeto a } \quad a(t)=r a(t)+y+g(t)-c(t)(1-\alpha i(t)), \\
\text { Hamiltoniano } \quad H[c(t), m(t), \lambda(t)]=u[c(t), m(t)] e^{-r t}+\lambda(t) \dot{a}(t), \\
\text { C.P.O. } \quad H_{c}=0, \quad H_{m}=0, \quad H_{\lambda}=\dot{a}(t) \quad \mathrm{y} \quad-H_{a}=\lambda(t) .
\end{array}\right. \\
& \left\{\begin{array}{l}
\text { Maximizar } \quad \int_{0}^{\infty} u[c(t), m(t)] e^{-r t} \mathrm{~d} t, \\
\text { sujeto a } \quad a(t)=r a(t)+y+g(t)-c(t)(1-\alpha i(t)), \\
\text { Hamiltoniano } H[c(t), m(t), \lambda(t)]=u[c(t), m(t)] e^{-r t}+\lambda(t) \dot{a}(t)+\lambda(t) a(t), \\
\text { C.P.O. } \quad H_{c}=0, \quad H_{m}=0, \quad H_{a}=0 \quad \mathrm{y} \quad H_{\lambda}=0 .
\end{array}\right.
\end{aligned}
$$


Francisco Venegas Martínez / Abigail Rodríguez Nava

\section{APÉNDICE A LA SECCIÓN 5}

En este apéndice se establecen dos resultados útiles en el desarrollo del modelo estocástico de estabilización temporal:

1) El lema de Itô para procesos combinados de difusión y saltos de Poisson, el cual puede ser enunciado de la siguiente manera. Dada la ecuación diferencial estocástica lineal y homogénea

$$
\mathrm{d} x_{t}=x_{t}\left(\alpha \mathrm{d} t+\sigma \mathrm{d} z_{t}+\gamma \mathrm{d} q_{t}\right), \quad z_{t} \sim N(0, t), \quad q_{t} \sim P(\lambda t)
$$

y una función $g\left(x_{t}\right)$ continua y dos veces diferenciable, entonces la diferencial estocástica de $g\left(x_{t}\right)$ está dada por

$\mathrm{d} g\left(x_{t}\right)=\left[g_{x}\left(x_{t}\right) \alpha x_{t}+\frac{1}{2} g_{x x}\left(x_{t}\right) \sigma^{2} x_{t}^{2}\right] \mathrm{d} t+g_{x}\left(x_{t}\right) \sigma x_{t} \mathrm{~d} z_{t}+\left[g\left(x_{t}(1+\gamma)\right)-g\left(x_{t}\right)\right] \mathrm{d} q_{t}$.

2) La solución a la ecuación anterior satisface

$$
x_{t}=x_{0} \exp \left\{\left(\alpha-\frac{1}{2} \sigma^{2}\right) t+\sigma \int_{0}^{t} \mathrm{~d} z_{u}+\log (1+\gamma) \int_{0}^{t} \mathrm{~d} q_{u}\right\}
$$

Es importante tener presente, al usar la expresión anterior, que para $t \geq 0$ las propiedades para $z_{t}$ y $q_{t}$ son:

$$
E\left[\int_{0}^{t} d z_{u}\right]=0, \quad E\left[\left(\int_{0}^{t} d z_{u}\right)^{2}\right]=\int_{0}^{t} d u=t \quad \mathrm{y} \quad E\left[\int_{0}^{t} d q_{u}\right]=\lambda t
$$

